Research Article

\title{
Thermodynamic Prescription of Cosmological Constant in the Randall-Sundrum II Brane
}

\author{
Tanwi Bandyopadhyay (D) \\ Adani Institute of Infrastructure Engineering, Ahmedabad-382421, India \\ Correspondence should be addressed to Tanwi Bandyopadhyay; tanwib@gmail.com
}

Received 13 June 2018; Revised 6 August 2018; Accepted 8 October 2018; Published 25 October 2018

Academic Editor: Elias C. Vagenas

Copyright (C) 2018 Tanwi Bandyopadhyay. This is an open access article distributed under the Creative Commons Attribution License, which permits unrestricted use, distribution, and reproduction in any medium, provided the original work is properly cited. The publication of this article was funded by SCOAP ${ }^{3}$

In this work, we apply the quantum corrected entropy function derived from the Generalized Uncertainty Principle (GUP) to the holographic equipartition law to study the cosmological scenario in the Randall-Sundrum (RS) II brane. An extra driving term has come up in the effective Friedmann equation for a homogeneous, isotropic, and spatially flat universe. Further, thermodynamic prescription of the universe constraints this term eventually with an order equivalent to that of the cosmological constant.

\section{Introduction}

In order to give an explanation of higher-dimensional theory, Randall and Sundrum $[1,2]$ proposed an idea of a bulk-brane model, where the four-dimensional world in which we live is called the 3-brane (a domain wall) that is embedded in a higher-dimensional space-time (bulk). According to the theory, the brane confines all the matter field; only gravity propagates in the bulk. Moreover the extra fifth dimension need not be finite; it can extend to infinity in either side of the brane. The concept of brane world scenarios shows a possibility to resolve the problem of unification of all forces and particles in nature. The main equations governing the cosmological evolutions of the brane differ from the corresponding Friedmann equations in standard cosmology [3-6]. The difference lies in the fact that the energy density of the brane appears to be in a quadratic form whereas, in standard cosmology, the energy density appears linearly in the field equations. This model is also consistent with string theory and may resolve the so-called hierarchy problem or the source of dark energy and dark matter $[7,8]$. The later theory is one of the overwhelming theories of the current era. The concept of dark matter had been first proposed $[9,10]$ in the context of studying galaxy clusters. The dark energy, on the other hand, is a completely new component which produces sufficient negative pressure. This drives the cosmic acceleration which has also been substantiated by the observational evidence over the years. The observational data clearly states that the current universe is flat having an approximate cosmic content of $21 \%$ dark matter, $72 \%$ dark energy, and the rest in the form of visible matter and radiation. All these imply that the standard cosmological models need to be modified with the models of dark matter and dark energy. Unfortunately, very little is known about dark energy. Hence there exist many prospective candidates for this cosmic component. Among them, cosmological constant $\Lambda$ is the most popular having an equation of state $p_{\Lambda}=-\rho_{\Lambda}$. This model is known as the $\Lambda$ CDM model (cold dark matter) [11-14]. This theory has a major drawback in terms of order of measurement. The observed value of $\Lambda$ is many orders of magnitude smaller than its theoretical value predicted in quantum field theory. This is termed as the cosmological constant problem and, to resolve this, one of the many proposed cosmological models is the varying cosmological constant $(\Lambda(t) \mathrm{CDM})$ model [1520].

On the other hand, one of the key features of quantum theory of gravity is called the holographic principle. This states that, in a bounded system, the number of degrees of freedom is associated with entropy and scales with the area enclosed [21-23]. Under this principle, gravity is shown to be 
an entropic force derived from the changes in the BekensteinHawking entropy [24-26]. Further, many studies focussed on derivation and investigation of the Friedmann and acceleration equations in the background of entropic cosmology [27-29]. Various forms of entropy have been applied in these studies [30-37]. In some of them, an extra driving term is derived from entropic forces on the horizon of the universe in order to explain its accelerated expansion. Intrigued by the holographic principle, very recently Padmanabhan [38] has proposed a different approach saying that the cosmic space is emergent as the cosmic time progresses. It has been termed the holographic equipartition law. According to this, the rate of expansion of the universe is related to the difference between the surface degrees of freedom on the holographic horizon and the bulk degrees of freedom inside. Keeping this in the background, the cosmological equations were derived and examined both in classical and in modified theories of gravity [39-45]. For most of these studies, the Bekenstein-Hawking entropy played the major role.

Very recently, a similar study has been carried out in [46], where a modified Rényi entropy was chosen instead of the Bekenstein-Hawking entropy and a constant-like term was obtained in the field equations. Imposing an analytical constraint, this term showed behavior similar to the varying cosmological constant. Further, the power-law corrected entropy was also tested in the same mechanism and similar results were found in [47]. This surely necessitates more investigation into the alternative studies of dark energy and the cosmological constant in modified gravity theories. We have followed this novel approach to study the underlying cosmological scenario in the RS II brane model considering the quantum corrected form of the entropy function derived from the Generalized Uncertainty Principle (GUP) [48]. A similar study has been carried out in [44] in Einstein's gravity but our entropy function is unique in its $\sqrt{\text { Area }}$ form. The necessity and motivation for choosing this entropy function was discussed later in detail. The GUP corrected entropy was applied to the holographic equipartition law in a fourdimensional universe embedded in a conformally flat fivedimensional space-time. Consequently, an analogous extra driving term is derived in the modified Friedmann equations. Further thermodynamical investigations showed that this extra term is of an order identical to the order of cosmological constant.

The paper is organized in the following way: In Section 2, we briefly review the $\Lambda(t) \mathrm{CDM}$ model and the modified field equations in the context of brane world gravity. In Section 3, the expansion of the cosmic space is treated as an emergent process and the modified Friedmann equations are retrieved from the holographic equipartition law in the absence of any dark energy component. Section 4 presents a brief review of GUP corrected entropy. Section 4.1 discusses the results of application of GUP corrected entropy into the holographic equipartition law. In Section 4.2, the validity of the Generalized Second Law of Thermodynamics (GSLT) is assumed and the behavior of the extra driving term is analyzed. Finally, a brief discussion on our study is made in Section 5 .

\section{Main Equations: $\Lambda(t)$ CDM Model in Brane World}

A homogeneous, isotropic, spatially flat FriedmannRobertson-Walker (FRW) universe in the natural unit system $\left(G=c=\hbar=k_{B}=1\right)$ is given by

$$
d s^{2}=d t^{2}-a^{2}(t)\left[d r^{2}+r^{2}\left(d \theta^{2}+\sin ^{2} \theta d \phi^{2}\right)\right]
$$

which is considered to be embedded in a conformally flat fivedimensional space-time. The form of the energy momentum tensor for a combination of dark matter and dark energy is

$$
T_{\mu}^{\nu}=\left(\rho_{m}+p_{m}+\rho_{\Lambda}+p_{\Lambda}\right) u_{\mu} u^{\nu}-\left(p_{m}+p_{\Lambda}\right) \delta_{\mu}^{\nu} .
$$

Generally a barotropic equation of state $p_{m}=\omega_{m} \rho_{m}$ is chosen for the matter part on the brane having energy density $\rho_{m}$ and pressure $p_{m}$ and a variable cosmological constant is chosen as the component of dark energy having energy density $\rho_{\Lambda}$ and pressure $p_{\Lambda}\left(=-\rho_{\Lambda}\right)$. The four-velocity $u_{\mu}$ in the comoving coordinate system takes the form $u_{\mu}=\delta_{\mu}{ }^{t}$. Thus the effective Einstein equations on the brane are [49]

$$
\frac{\dot{a}^{2}}{a^{2}}=H^{2}=\frac{8 \pi}{3}\left[\rho_{T}\left(1+\frac{\rho_{T}}{2 \lambda}\right)\right]
$$

and

$$
\begin{aligned}
\frac{\ddot{a}}{a} & =\dot{H}+H^{2} \\
& =-\frac{4 \pi}{3}\left[\rho_{T}\left(1+\frac{2 \rho_{T}}{\lambda}\right)+3 p_{\Lambda}\left(1+\frac{\rho_{T}}{\lambda}\right)\right],
\end{aligned}
$$

where $\rho_{T}=\rho_{m}+\rho_{\Lambda}$ is the total energy density, $p_{T}=p_{m}+$ $p_{\Lambda}$ is the total pressure, $\lambda$ is the positive brane tension, the Hubble parameter is given by $H(t)=\dot{a} / a$, and $\mathrm{a}(\mathrm{t})$ is the scale factor in the flat FRW brane model.

Equations (3) and (4) can be explicitly written as

$$
\frac{\dot{a}^{2}}{a^{2}}=\frac{8 \pi}{3} \rho_{m \mathrm{eff}}+\frac{1}{3} \Lambda(t)_{\mathrm{eff}}
$$

and

$$
\begin{aligned}
\frac{\ddot{a}}{a} & =-\frac{4 \pi}{3}\left[\left(\rho_{m}+3 p_{m}\right)\right. \\
& \left.+\frac{1}{\lambda}\left(2 \rho_{m}+3 p_{m}+2 \rho_{m} \rho_{\Lambda}+3 \rho_{m} p_{m}\right)\right]+\frac{1}{3} \Lambda(t)_{\mathrm{eff}},
\end{aligned}
$$

where $\rho_{m \text { eff }}=\rho_{m}\left(1+\rho_{m} / 2 \lambda\right)$ and $\Lambda(t)_{\text {eff }}=8 \pi\left[\rho_{\Lambda}\left(1+\rho_{\Lambda} / 2 \lambda+\right.\right.$ $\left.\left.\rho_{m} / \lambda\right)\right]$.

For the present brane model with matter field given by (2), the explicit form of the energy momentum conservation relation $\left(T_{\mu}{ }^{\nu} ; \nu=0\right)$ is

$$
\dot{\rho_{m}}+3 \frac{\dot{a}}{a}\left(\rho_{m}+p_{m}\right)=-\dot{\rho_{\Lambda}} \simeq-\frac{\Lambda(\dot{t})_{\mathrm{eff}}}{8 \pi} .
$$

Instead of a variable $\rho_{\Lambda}$, if we choose a constant $\rho_{\Lambda}$, then the field equations together with the continuity equation will be identical to the corresponding equations in the standard $\Lambda$ CDM model. 


\section{Field Equations Derived from the Holographic Equipartition Law}

For a pure de Sitter universe with Hubble parameter $H$, the holographic principle can be described by the relation [38]

$$
N_{\text {sur }}=N_{\text {bulk }}
$$

where $N_{\text {sur }}$ denotes the number of the degrees of freedom on the holographic screen with Hubble radius $r_{H}=1 / H$ :

$$
N_{\text {sur }}=\frac{4 \pi}{H^{2}}=4 S_{H} \text {. }
$$

Here $S_{H}$ is the entropy on the Hubble horizon. The number of degrees of freedom in bulk is said to obey the equipartition law of energy

$$
N_{\text {bulk }}=\frac{2|E|}{T} \text {. }
$$

In the context of brane world models, the induced active gravitational mass on the brane $|M|=|E|$ has the form [50]

$$
\begin{aligned}
& |M|=\frac{4 \pi}{3 H^{3}}\left|\left(\rho_{T}+3 p_{T}+\frac{3 \rho_{T} p_{T}}{\lambda}+\frac{2 \rho_{T}^{2}}{\lambda}\right)\right|=-\epsilon \\
& \cdot \frac{4 \pi}{3 H^{3}}\left\{\left[\left(\rho_{m}+3 p_{m}\right)\right.\right. \\
& \left.+\frac{1}{\lambda}\left(2 \rho_{m}+3 p_{m}+2 \rho_{m} \rho_{\Lambda}+3 \rho_{m} p_{m}\right)\right]+\frac{1}{4 \pi} \\
& \left.\cdot \Lambda(t)_{\mathrm{eff}}\right\}
\end{aligned}
$$

for the choice of the matter field (2). The parameter $\epsilon$ is defined later. Using the above expression of $|M|$ and the horizon temperature $T=H / 2 \pi$, we get the expression of $N_{\text {bulk }}$ as

$$
\begin{aligned}
& N_{\text {bulk }}=-\epsilon \frac{16 \pi^{2}}{3 H^{4}}\left\{\left[\left(\rho_{m}+3 p_{m}\right)\right.\right. \\
& \left.+\frac{1}{\lambda}\left(2 \rho_{m}+3 p_{m}+2 \rho_{m} \rho_{\Lambda}+3 \rho_{m} p_{m}\right)\right]+\frac{1}{4 \pi} \\
& \left.\cdot \Lambda(t)_{\text {eff }}\right\} .
\end{aligned}
$$

Since the real world is not purely but asymptotically de Sitter, therefore one may propose that the expansion rate of the cosmic volume is related to the difference of these two degrees of freedom. The analytical form of this is described as [38]

$$
\frac{d V}{d t}=l_{p}^{2}\left(N_{\text {sur }}-\epsilon N_{\text {bulk }}\right) .
$$

Equation (13) is known as the holographic equipartition law. Here $V=4 \pi / 3 H^{3}$ is the cosmic volume and the parameter $\epsilon$ is defined by $[38,51]$

$$
\equiv\left\{\begin{array}{l}
+1, \quad \text { when }\left[\left(\rho_{m}+3 p_{m}\right)+\frac{1}{\lambda}\left(3 p_{m}+2 \rho_{m}+3 \rho_{m} p_{m}\right)\right]<0 \\
-1, \quad \text { when }\left[\left(\rho_{m}+3 p_{m}\right)+\frac{1}{\lambda}\left(3 p_{m}+2 \rho_{m}+3 \rho_{m} p_{m}\right)\right]>0 .
\end{array}\right.
$$

Here, we have considered that there is no dark energy component in the 3-brane, i.e., $\Lambda(t)_{\text {eff }} \sim \rho_{\Lambda}=0$. In this case $\left[\left(\rho_{m}+3 p_{m}\right)+(1 / \lambda)\left(3 p_{m}+2 \rho_{m}+3 \rho_{m} p_{m}\right)\right]<0$ for the acceleration of the universe. Hence from (11) and (14), the definition of the parameter $\epsilon$ is well justified.

One can write from (9), (12), and (13)

$$
\begin{gathered}
-4 \pi \frac{\dot{H}}{H^{4}}=\left\{4 S_{H}+\frac{16 \pi^{2}}{3 H^{4}}\left[\left(\rho_{m}+3 p_{m}\right)\right.\right. \\
\left.\left.+\frac{1}{\lambda}\left(3 p_{m}+2 \rho_{m}+3 \rho_{m} p_{m}\right)\right]\right\}
\end{gathered}
$$

or equivalently

$$
\begin{aligned}
\dot{H}= & -\frac{4 \pi}{3}\left[\left(\rho_{m}+3 p_{m}\right)+\frac{1}{\lambda}\left(3 p_{m}+2 \rho_{m}+3 \rho_{m} p_{m}\right)\right] \\
& -\frac{H^{4} S_{H}}{\pi} .
\end{aligned}
$$

The acceleration equation is therefore read as

$$
\begin{aligned}
\frac{\ddot{a}}{a}= & -\frac{4 \pi}{3}\left[\left(\rho_{m}+3 p_{m}\right)+\frac{1}{\lambda}\left(3 p_{m}+2 \rho_{m}+3 \rho_{m} p_{m}\right)\right] \\
& +H^{2}\left(1-\frac{H^{2} S_{H}}{\pi}\right) .
\end{aligned}
$$

Thus we have derived the acceleration equation from the holographic equipartition law and an extra driving term appears on the right side of the equation. This term vanishes when one chooses the Bekenstein-Hawking entropy for $S_{H}$. The acceleration equation will then be

$$
\frac{\ddot{a}}{a}=-\frac{4 \pi}{3}\left[\left(\rho_{m}+3 p_{m}\right)+\frac{1}{\lambda}\left(3 p_{m}+2 \rho_{m}+3 \rho_{m} p_{m}\right)\right]
$$

which is identical to (6) with $\Lambda(t)_{\text {eff }} \sim \rho_{\Lambda}=0$. Hence in this case, the field equation and the corresponding energy conservation equation become

$$
\frac{\dot{a}^{2}}{a^{2}}=\frac{8 \pi G}{3} \rho_{m \mathrm{eff}}
$$

and

$$
\dot{\rho}_{m}+3 \frac{\dot{a}}{a}\left(\rho_{m}+p_{m}\right)=0 .
$$

However, any other form of $S_{H}$ will not result in the above set of equations and the cosmological implications will definitely be something else.

\section{GUP Corrected Entropy on the Horizon}

In recent years, a number of studies in general relativity and modified gravity theories came to surface due to the discovery of different aspects of black hole solutions. Black holes are thermodynamic objects with well-defined entropy. Generally, the Bekenstein-Hawking entropy [52-54]

$$
S_{B H}=\frac{A}{4 l_{p}^{2}}
$$


is chosen for the same. Here $A$ is the surface area of the sphere with the Hubble horizon $r_{H}=1 / H$ and $l_{p}=$ $\sqrt{G \hbar / c^{3}} \simeq 10^{-35} \mathrm{~m}$ is the Planck length. With $A=4 \pi r_{H}{ }^{2}$, we can write

$$
S_{B H}=\frac{\pi r_{H}^{2}}{l_{p}^{2}} .
$$

Instead of a flat universe, if we choose a nonflat universe, then the apparent horizon $r_{A}=1 / \sqrt{H^{2}+k / a^{2}}$ should be used as the horizon radius instead of the Hubble horizon. Corrections in this entropy formula were needed to accommodate the newly emerging physics from string theory and loop quantum gravity (LQG). Several of these theories predicted quantum corrections to the entropy-area relation [55-64]

$$
S_{\mathrm{QG}}=\frac{A}{4 l_{p}^{2}}+C_{0} \ln \left(\frac{A}{4 l_{p}^{2}}\right)+\sum_{n=1}^{\infty} C_{n}\left(\frac{A}{4 l_{p}^{2}}\right)^{-n},
$$

where the coefficients $C_{n}$ are model dependent parameters. Recent rigorous calculations from LQG have fixed the value of $C_{0}=-1 / 2$ [59]. On the other hand, Mead [65] first pointed out that the Heisenberg uncertainty principle could be affected by gravity. Later, a considerable amount of effort had been put to the modified commutation relations between position and momenta commonly known as the Generalized Uncertainty Principle (GUP) from different perspectives of quantum aspects of gravity. All these studies eventually led to the GUP corrected entropy form [66-69]

$$
\begin{aligned}
S_{G U P}= & \frac{A}{4 l_{p}{ }^{2}}+\frac{\sqrt{\pi} \alpha_{0}}{4} \sqrt{\frac{A}{4 l_{p}{ }^{2}}}-\frac{\pi \alpha_{0}{ }^{2}}{64} \ln \left(\frac{A}{4 l_{p}{ }^{2}}\right) \\
& +O\left(l_{p}^{3}\right) .
\end{aligned}
$$

Here $\alpha_{0}$ is a dimensionless constant prescribed in the deformed commutation relations [70]. The leading contribution of this new entropy function lies in its second term $\sim \sqrt{\text { Area }}$. This is an extra term to the already existing logarithmic correction to entropy derived from the quantum gravity effects. Due to the difference in the leading order correction term, the underlying nature of such model needs to be investigated in four-dimensional Einstein's gravity as well as in higher-dimensional modified theories of gravity. Based on many similarities between the black hole horizon and cosmological horizon and on the assumption that the universe should be described by the quantum language, we employ this newly obtained GUP corrected entropy of the black hole horizon as the entropy of the cosmological horizon in the natural unit system

$$
S_{\mathrm{Q}}=\frac{A}{4}+\frac{\sqrt{\pi} \alpha_{0}}{4} \sqrt{\frac{A}{4}}-\frac{\pi \alpha_{0}^{2}}{64} \ln \left(\frac{A}{4}\right)
$$

which on further calculation becomes

$$
S_{\mathrm{Q}}=S_{B H}\left[1+\frac{\alpha_{0} H}{4}-\frac{\alpha_{0}^{2} H^{2}}{64} \ln \left(\frac{\pi}{H^{2}}\right)\right] .
$$

Here $S_{B H}=\pi / H^{2}$. The novelty of this expression is that when $\alpha_{0}=0$, then $S_{Q}$ becomes $S_{B H}$.
4.1. Consequences of GUP Corrected Entropy into the Holographic Equipartition Law. Here, we apply the GUP corrected entropy function $S_{\mathrm{Q}}$ into the holographic equipartition law; i.e., we consider that

$$
S_{H}=S_{\mathrm{Q}}=S_{B H}\left[1+\frac{\alpha_{0} H}{4}-\frac{\alpha_{0}^{2} H^{2}}{64} \ln \left(\frac{\pi}{H^{2}}\right)\right] .
$$

Substituting this new form of $S_{H}$ in (17), we have

$$
\begin{aligned}
\frac{\ddot{a}}{a}= & -\frac{4 \pi}{3}\left[\left(\rho_{m}+3 p_{m}\right)+\frac{1}{\lambda}\left(3 p_{m}+2 \rho_{m}+3 \rho_{m} p_{m}\right)\right] \\
& +\left[\frac{\alpha_{0}^{2} H^{4}}{64} \ln \left(\frac{\pi}{H^{2}}\right)-\frac{\alpha_{0} H^{3}}{4}\right] .
\end{aligned}
$$

The extra driving term appearing on the right side of the equation needs to be positive for the current cosmic acceleration.

In the brane world gravity, the field equations together with the continuity equation then become

$$
\begin{aligned}
\frac{\dot{a}^{2}}{a^{2}}= & \frac{8 \pi}{3} \rho_{m \text { eff }}+f_{\alpha}(H) \\
\frac{\ddot{a}}{a}= & -\frac{4 \pi}{3}\left[\left(\rho_{m}+3 p_{m}\right)+\frac{1}{\lambda}\left(2 \rho_{m}+3 p_{m}+3 \rho_{m} p_{m}\right)\right] \\
& +f_{\alpha}(H)
\end{aligned}
$$

and

$$
\dot{\rho_{m}}+3 \frac{\dot{a}}{a}\left(\rho_{m}+p_{m}\right)=-\frac{\left.3 f_{\alpha} \dot{(} H\right)}{8 \pi},
$$

where the extra term $f_{\alpha}(H)$ is given by

$$
f_{\alpha}(H)=\frac{\alpha_{0}^{2} H^{4}}{64} \ln \left(\frac{\pi}{H^{2}}\right)-\frac{\alpha_{0} H^{3}}{4} .
$$

Let us now discuss the evolution of this extra driving term from entropy function (27) and acceleration equation (30). Equation (30) is the final equation incorporating all three corrections. As $S_{B H}$ is positive, hence the following restriction is to be obeyed by the parameters for $S_{Q}$ to be positive:

$$
\left[\frac{\alpha_{0} H}{16} \ln \left(\frac{\pi}{H^{2}}\right)-1\right]<\frac{4}{\alpha_{0} H} .
$$

Again for the current cosmic acceleration

$$
0<f_{\alpha}(H)=\frac{\alpha_{0} H^{3}}{4}\left[\frac{\alpha_{0} H}{16} \ln \left(\frac{\pi}{H^{2}}\right)-1\right] .
$$

Hence it is clear from (33) and (34) that

$$
f_{\alpha}(H)<H^{2} .
$$

A similar constraint can be derived from the study of the Generalized Second Law of Thermodynamics (GSLT) as presented in the following subsection. 
4.2. Generalized Second Law of Thermodynamics (GSLT). Here we shall discuss the GSLT in the current prescription. Considering $S_{T}$ as the total entropy of the universe, one can write

$$
\dot{S_{T}}=\dot{S_{\mathrm{Q}}}+\dot{S_{I}},
$$

where $S_{I}$ is the entropy of matter inside the horizon. From (27), we can write

$$
\dot{S_{\mathrm{Q}}}=\dot{S_{B H}}\left[1-\left(\frac{\alpha_{0}^{2} H^{2}}{64}-\frac{\alpha_{0} H}{8}\right)\right],
$$

where

$$
S_{B H}^{\cdot}=\frac{d}{d t}\left(\frac{\pi}{H^{2}}\right)=-\frac{2 \pi \dot{H}}{H^{3}} .
$$

Since $\dot{S_{B H}}>0$, to satisfy $\dot{S_{Q}}>0$, the following restriction needs to be obeyed:

$$
\left(\frac{\alpha_{0}^{2} H^{2}}{64}-\frac{\alpha_{0} H}{8}\right)<1 .
$$

In order to obtain the rate of change of entropy of the matter inside the horizon, we consider the Gibbs equation $[71,72]$

$$
T_{I} d S_{I}=d E_{I}+p_{T} d V
$$

where $V$ is the volume inside the horizon and $E_{I}=\rho_{T} d V$ stands for the internal energy. The temperature of the matter $T_{I}$ inside the horizon has been assumed to be equivalent to the horizon temperature $T=H / 2 \pi$. In absence of any dark energy component, this equation takes the form

$$
\begin{aligned}
T \dot{S}_{I} & =\left[\dot{\rho_{m}}+3 \frac{\dot{a}}{a}\left(\rho_{m}+p_{m}\right)\right] V \\
& =-\frac{3 f_{\alpha}(\dot{H}) V}{8 \pi}
\end{aligned}
$$

where we have used modified continuity equation (31) to obtain the expression of $\dot{S}_{I}$. Taking time derivative of (32) and using the expression of horizon temperature $T$, one can yield

$$
\dot{S}_{I}=S_{B H}\left[\frac{\alpha_{0}{ }^{2} H^{2}}{32} \ln \left(\frac{\pi}{H^{2}}\right)-\frac{3 \alpha_{0} H}{8}-\frac{\alpha_{0}{ }^{2} H^{2}}{64}\right] .
$$

Thus from (37) and (42), the rate of change of total entropy of the universe becomes

$$
\begin{aligned}
& \dot{S_{T}} \\
& =S_{B H}^{\cdot}\left[1-\left\{\frac{\alpha_{0} H}{4}+\frac{\alpha_{0}{ }^{2} H^{2}}{32}-\frac{\alpha_{0}{ }^{2} H^{2}}{32} \ln \left(\frac{\pi}{H^{2}}\right)\right\}\right] .
\end{aligned}
$$

Again as $\dot{S_{B H}}>0$, to satisfy $\dot{S_{T}}>0$, the following condition must be attained:

$$
\left[\frac{\alpha_{0} H}{4}+\frac{\alpha_{0}{ }^{2} H^{2}}{32}-\frac{\alpha_{0}{ }^{2} H^{2}}{32} \ln \left(\frac{\pi}{H^{2}}\right)\right]<1 .
$$

From (39) and (44), one can easily derive

$$
f_{\alpha}(H)>\frac{H^{2}}{2} \text {. }
$$

Thus, we attain a very interesting result from (35) and (45)

$$
\frac{H^{2}}{2}<f_{\alpha}(H)<H^{2}
$$

Following the arguments of [47] as for the observational constraint $\dot{H}<0$ [73], one can assume $H_{0}$ to be the minimum value for $H$ and arrive at a stricter constraint

$$
\begin{aligned}
\frac{H_{0}{ }^{2}}{2} & <f_{\alpha}(H)<H_{0}{ }^{2} \\
& \Longrightarrow O\left(f_{\alpha}(H)\right) \lesssim O\left(H_{0}^{2}\right) .
\end{aligned}
$$

This result is analogous to the one presented in both [46, 47], though, in the former study, a mathematical condition was imposed to obtain similar restriction while, in the latter, it evolved through the validity of the GSLT. Further probing into the standard $\Lambda$ CDM model, we obtain $\Lambda=3 H_{0}{ }^{2} \Omega_{\Lambda}$. This implies that

$$
O\left(\frac{\Lambda}{3}\right)=O\left(H_{0}^{2} \Omega_{\Lambda}\right)
$$

As from the Planck (2015) results [14], $\Omega_{\Lambda}=0.692$, which is of order one. This yields to

$$
O\left(\frac{\Lambda}{3}\right) \simeq O\left(H_{0}^{2}\right)
$$

Thus the order of the extra driving term in the acceleration equation becomes equivalent to the order of the cosmological constant term. This result however seems to be model-independent as the positive brane tension did not play any significant role in deriving the analogy.

\section{Discussions}

In the present work, our aim was to study the cosmic evolution in the brane world gravity with the help of the holographic equipartition law. We have applied the quantum corrected form of the entropy function derived from the Generalized Uncertainty Principle in the holographic equipartition law to derive the modified cosmological equations in a homogeneous, isotropic, and spatially flat 3-brane embedded in a five-dimensional bulk. The novelty of the study lies in the $\sqrt{\text { Area }}$ form of the entropy function. It was noticed that the acceleration equation contains an extra driving term of an order consistent with the order of the cosmological constant. A similar constraint was obtained assuming the validity of GSLT. The study remained to be model-independent and the positive brane tension did not play any crucial role for the attained result. However, it should be understood that our aim was not to verify the GSLT in the modified gravity theory. Rather we were interested in the evolution of the extra driving term appearing in the acceleration equation due to 
imposition of the holographic equipartition law for a specific GUP corrected entropy function whose leading order term is different from the existing forms. This may shed new light on the studies of the cosmological constant problem in modified gravity theories.

\section{Data Availability}

The data used to support the findings of this study are included within the article.

\section{Conflicts of Interest}

The author declares that they have no conflicts of interest.

\section{Acknowledgments}

The author is thankful to IUCAA, Pune, for their warm hospitality and excellent research facilities where part of the work has been done during a visit under the Associateship Programme.

\section{References}

[1] L. Randall and R. Sundrum, "Large mass hierarchy from a small extra dimension," Physical Review Letters, vol. 83, no. 17, pp. 3370-3373, 1999.

[2] L. Randall and R. Sundrum, "An alternative to compactification," Physical Review Letters, vol. 83, no. 23, pp. 4690-4693, 1999.

[3] P. Binétruy, C. Deffayet, U. Ellwanger, and D. Langlois, "Brane cosmological evolution in a bulk with cosmological constant," Physics Letters B, vol. 477, pp. 285-291, 2000.

[4] P. Binétruy, C. Deffayet, and D. Langlois, "Non-conventional cosmology from a brane universe," Nuclear Physics, B, vol. 565, no. 1-2, pp. 269-287, 2000.

[5] J. Ponce De Leon, "Variation of G, $\Lambda(4)$ and Vacuum Energy from Brane-World Models," Modern Physics Letters A, vol. 17, no. 37, pp. 2425-2441, 2002.

[6] J. Ponce De Leon, "Equivalence between space-time-matter and brane-world theories," Modern Physics Letters A, vol. 16, no. 35, pp. 2291-2303, 2001.

[7] A. Lukas, B. A. Ovrut, K. S. Stelle, and D. Waldram, "Universe as a domain wall," Physical Review D: Particles, Fields, Gravitation and Cosmology, vol. 59, no. 8, 086001, 9 pages, 1999.

[8] A. Lukas, B. A. Ovrut, and D. Waldram, "Cosmological solutions of Hořava-Witten theory," Physical Review D: Particles, Fields, Gravitation and Cosmology, vol. 60, no. 8, Article ID 086001, 1999.

[9] F. Zwicky, "Die Rotverschiebung von extragalaktischen Nebeln," Helvetica Physica Acta, vol. 6, pp. 110-127, 1933.

[10] F. Zwiecky, "Nebulae as Gravitational Lenses," Physical Review Journals, vol. 51, p. 290, 1937.

[11] S. Perlmutter, G. Aldering, M. Della Valle et al., "Discovery of a supernova explosion at half the age of the Universe," Nature, vol. 391, no. 6662, pp. 51-54, 1998.

[12] A. G. Riess, A. V. Filippenko, and P. Challis, "Observational evidence from supernovae for an accelerating universe and a cosmological constant," The Astronomical Journal, vol. 116, p. 1009, 1998.
[13] A. G. Riess, L.-G. Strolger, and S. Casertano, "New Hubble Space Telescope Discoveries of Type Ia Supernovae at $z \geq 1$ : Narrowing Constraints on the Early Behavior of Dark Energy," The Astrophysical Journal, vol. 659, no. 1, p. 98, 2007.

[14] P. A. R. Ade et al., "Planck 2015 results - XIII. Cosmological parameters," Astronomy \& Astrophysics (A\&A), vol. 594, Article ID A13, 63 pages, 2016.

[15] I. L. Shapiro and J. Solà, "The scaling evolution of the cosmological constant," Journal of High Energy Physics, no. 02, Article ID 006, 2002.

[16] S. Basilakos, M. Plionis, and J. Solà, "Hubble expansion and structure formation in time varying vacuum models," Physical Review D: Particles, Fields, Gravitation and Cosmology, vol. 80, no. 8, Article ID 083511, 2009.

[17] J. P. Mimoso and D. Pavón, "Entropy evolution of universes with initial and final de Sitter eras," Physical Review D: Particles, Fields, Gravitation and Cosmology, vol. 87, Article ID 047302, 2013.

[18] M. H. P. M. van Putten, "Accelerated expansion from cosmological holography," Monthly Notices of the Royal Astronomical Society: Letters, vol. 450, no. 1, pp. L48-L51, 2015.

[19] A. Gómez-Valent, E. Karimkhani, and J. Solà, "Background history and cosmic perturbations for a general system of self-conserved dynamical dark energy and matter," Journal of Cosmology and Astroparticle Physics, vol. 12, Article ID 048, 2015.

[20] J. A. Lima, S. Basilakos, and J. Solà, "Nonsingular decaying vacuum cosmology and entropy production," General Relativity and Gravitation, vol. 47, no. 4, article 40, 2015.

[21] G. 't Hooft, "Dimensional Reduction in Quantum Gravity," Salamfest, pp. 0284-296, 1993.

[22] L. Susskind, "A predictive Yukawa unified SO(10) model: higgs and sparticle masses," Journal of Mathematical Physics, vol. 36, no. 7, article 139, pp. 6377-6396, 1995.

[23] R. Bousso, “The holographic principle," Reviews of Modern Physics, vol. 74, no. 3, pp. 825-874, 2002.

[24] T. Padmanabhan, "Equipartition of energy in the horizon degrees of freedom and the emergence of gravity," Modern Physics Letters A, vol. 25, no. 14, pp. 1129-1136, 2010.

[25] T. Padmanabhan, "Thermodynamical Aspects of Gravity: New insights," Reports on Progress in Physics, vol. 73, Article ID 046901, 2010.

[26] E. Verlinde, "On the origin of gravity and the laws of Newton," Journal of High Energy Physics, vol. 2011, 29 pages, 2011.

[27] A. Sheykhi, "Entropic corrections to Friedmann equations," Physical Review D: Particles, Fields, Gravitation and Cosmology, vol. 81, no. 10, Article ID 104011, 2010.

[28] A. Sheykhi and S. H. Hendi, "Power-law entropic corrections to Newton's law and Friedmann equations," Physical Review D: Particles, Fields, Gravitation and Cosmology, vol. 84, no. 4, 2011.

[29] S. Mitra, S. Saha, and S. Chakraborty, "Modified Hawking temperature and entropic force: a prescription in FRW model," Modern Physics Letters A, vol. 30, no. 13, 1550058 pages, 2015.

[30] D. A. Easson, P. H. Frampton, and G. F. Smoot, "Entropic accelerating universe," Physics Letters B, vol. 696, no. 3, pp. 273277, 2011.

[31] D. A. Easson, P. H. Frampton, and G. F. Smoot, "Entropic inflation," International Journal of Modern Physics A, vol. 27, Article ID 1250066, 2012.

[32] Y. F. Cai, J. Liu, and H. Li, "Entropic cosmology: a unified model of inflation and late-time acceleration," Physics Letters B, vol. 690, no. 3, pp. 213-219, 2010. 
[33] T. S. Koivisto, D. F. Motaá, and M. Zumalacárregui, “Constraining entropic cosmology," Journal of Cosmology and Astroparticle Physics, vol. 02, p. 027, 2011.

[34] N. Komatsu and S. Kimura, "Non-adiabatic-like accelerated expansion of the late universe in entropic cosmology," Physical Review D: Particles, Fields, Gravitation and Cosmology, vol. 87, Article ID 043531, 2013.

[35] N. Komatsu and S. Kimura, "Evolution of the universe in entropic cosmologies via different formulations," Physical Review D: Particles, Fields, Gravitation and Cosmology, vol. 89, Article ID 123501, 2014.

[36] M. P. Dabrowski and H. Gohar, "Abolishing the maximum tension principle," Physics Letters B, vol. 748, pp. 428-431, 2015.

[37] C. Tsallis and L. J. L. Cirto, "Black hole thermodynamical entropy," The European Physical Journal C, vol. 73, no. 7, p. 2487, 2013.

[38] T. Padmanabhan, "Emergence and Expansion of Cosmic Space as due to the Quest for Holographic Equipartition," https:// arxiv.org/abs/1206.4916.

[39] R. G. Cai, "Emergence of Space and Spacetime Dynamics of Friedmann-Robertson-Walker Universe," https://arxiv.org/abs/ 1207.0622 .

[40] K. Yang, Y.-X. Liu, and Y.-Q. Wang, "Emergence of Cosmic Space and the Generalized Holographic Equipartition," Physical Review D, vol. 86, Article ID 104013, 8 pages, 2012.

[41] Y. Ling and W.-J. Pan, "Note on the emergence of cosmic space in modified gravities," Physical Review D, vol. 88, Article ID 043518, 2013.

[42] A. F. Ali, "Emergence of cosmic space and minimal length in quantum gravity," Physics Letters B, vol. 732, pp. 335-342, 2014.

[43] T. Padmanabhan, "Emergent gravity paradigm: recent progress," Modern Physics Letters A, vol. 30, no. 3-4, 1540007, 21 pages, 2015.

[44] X.-X. Zeng and Y. Chen, "Quantum gravity corrections to fermions' tunnelling radiation in the Taub-NUT spacetime," General Relativity and Gravitation, vol. 47, no. 4, article 47, 2015.

[45] H. Moradpour and A. Sheykhi, "From the Komar mass and entropic force scenarios to the Einstein field equations on the Ads brane," International Journal of Theoretical Physics, vol. 55, no. 2, pp. 1145-1155, 2016.

[46] N. Komatsu, "Cosmological model from the holographic equipartition law with a modified Rényi entropy," The European Physical Journal C, vol. 77, no. 229, 2017.

[47] N. Komatsu, "Thermodynamic constraints on a varying cosmological-constant-like term from the holographic equipartition law with a power-law corrected entropy," Physical Review D, vol. 96, Article ID 103507, 2017.

[48] P. Vergueno and E. C. Vagenas, "Semiclassical corrections to black hole entropy and the generalized uncertainty principle," Physics Letters B, vol. 742, pp. 15-18, 2015.

[49] T. Bandyopadhyay, A. Baveja, and S. Chakraborty, "Gravitational collapse in the context of brane world scenario with decaying vacuum energy," Modern Physics Letters A, vol. 23, no. 9, pp. 685-693, 2008.

[50] Y. Ling and J. P. Wu, "A note on entropic force and brane cosmology," Journal of Cosmology and Astroparticle Physics, vol. 017, p. 1008, 2010.

[51] T. Padmanabhan, "Emergent perspective of gravity and dark energy," Research in Astronomy and Astrophysics, vol. 12, p. 891, 2012.
[52] J. D. Bekenstein, "Black holes and entropy," Physical Review D: Particles, Fields, Gravitation and Cosmology, vol. 7, pp. 23332346, 1973.

[53] J. D. Bekenstein, "Generalized second law of thermodynamics in black-hole physics," Physical Review D: Particles, Fields, Gravitation and Cosmology, p. 3292, 1974.

[54] J. D. Bekenstein, "Statistical black hole thermodynamics," Physical Review D: Particles, Fields, Gravitation and Cosmology, vol. 12, no. 10, pp. 3077-3085, 1975.

[55] R. K. Kaul and P. Majumdar, "Logarithmic correction to the Bekenstein-Hawking entropy," Physical Review Letters, vol. 84, no. 23, pp. 5255-5257, 2000.

[56] A. Ghosh and P. Mitra, "An improved estimate of black hole entropy in the quantum geometry approach," Physics Letters B, vol. 616, no. 1-2, pp. 114-117, 2005.

[57] A. J. Medved and E. C. Vagenas, "When conceptual worlds collide: the generalized uncertainty principle and the BekensteinHawking entropy," Physical Review D: Particles, Fields, Gravitation and Cosmology, vol. 70, no. 12, Article ID 124021, 5 pages, 2004.

[58] G. Amelino-Camelia, M. Arzano, and A. Procaccini, "Severe constraints on the loop-quantum-gravity energy-momentum dispersion relation from the black-hole area-entropy law," Physical Review D: Particles, Fields, Gravitation and Cosmology, vol. 70, no. 10, Article ID 107501, 2004.

[59] K. A. Meissner, "Black-hole entropy in loop quantum gravity," Classical and Quantum Gravity, vol. 21, no. 22, pp. 5245-5251, 2004.

[60] S. Das, P. Majumdar, and R. K. Bhaduri, "General logarithmic corrections to black-hole entropy," Classical and Quantum Gravity, vol. 19, no. 9, pp. 2355-2367, 2002.

[61] Y. S. Myung, "Logarithmic corrections to three-dimensional black holes and de Sitter spaces," Physics Letters B, vol. 579, no. 1-2, pp. 205-210, 2004.

[62] M. Domagala and J. Lewandowski, "Black-hole entropy from quantum geometry," Classical and Quantum Gravity, vol. 21, no. 22, pp. 5233-5243, 2004.

[63] A. Chatterjee and P. Majumdar, "Universal canonical black hole entropy," Physical Review Letters, vol. 92, no. 14, Article ID 141301, 2004.

[64] M. M. Akbar and S. Das, "Entropy corrections for Schwarzschild and Reissner Nordström black holes," Classical and Quantum Gravity, vol. 21, no. 6, pp. 1383-1392, 2004.

[65] C. A. Mead, "Possible Connection Between Gravitation and Fundamental Length," Physical Review D, vol. 135, Article ID B849, 1964.

[66] B. Majumder, "Black hole entropy and the modified uncertainty principle: a heuristic analysis," Physics Letters B, vol. 703, no. 4, pp. 402-405, 2011.

[67] R. J. Adler, P. Chen, and D. I. Santiago, "The generalized uncertainty principle and black hole remnants," General Relativity and Gravitation, vol. 33, no. 12, pp. 2101-2108, 2001.

[68] G. Amelino-Camelia, M. Arzano, Y. Ling, and G. Mandanici, "Black-hole thermodynamics with modified dispersion relations and generalized uncertainty principles," Classical and Quantum Gravity, vol. 23, no. 7, pp. 2585-2606, 2006.

[69] B. Majumder, "Black hole entropy with minimal length in tunneling formalism," General Relativity and Gravitation, vol. 45, no. 11, pp. 2403-2414, 2013.

[70] S. Das and E. C. Vagenas, "Universality of quantum gravity corrections," Physical Review Letters, vol. 101, Article ID 221301, 4 pages, 2008. 
[71] B. Wang, Y. G. Gong, and E. Abdalla, "Thermodynamics of an accelerated expanding universe," Physical Review D: Particles, Fields, Gravitation and Cosmology, vol. 74, Article ID 083520, 2006.

[72] G. Izquierdo and D. Pavon, "Dark energy and the generalized second law," Physics Letters B, vol. 633, no. 4-5, pp. 420-426, 2006.

[73] P. B. Krishna and T. Mathew, "Holographic equipartition and the maximization of entropy," Physical Review D, vol. 96, Article ID 063513, 2017. 

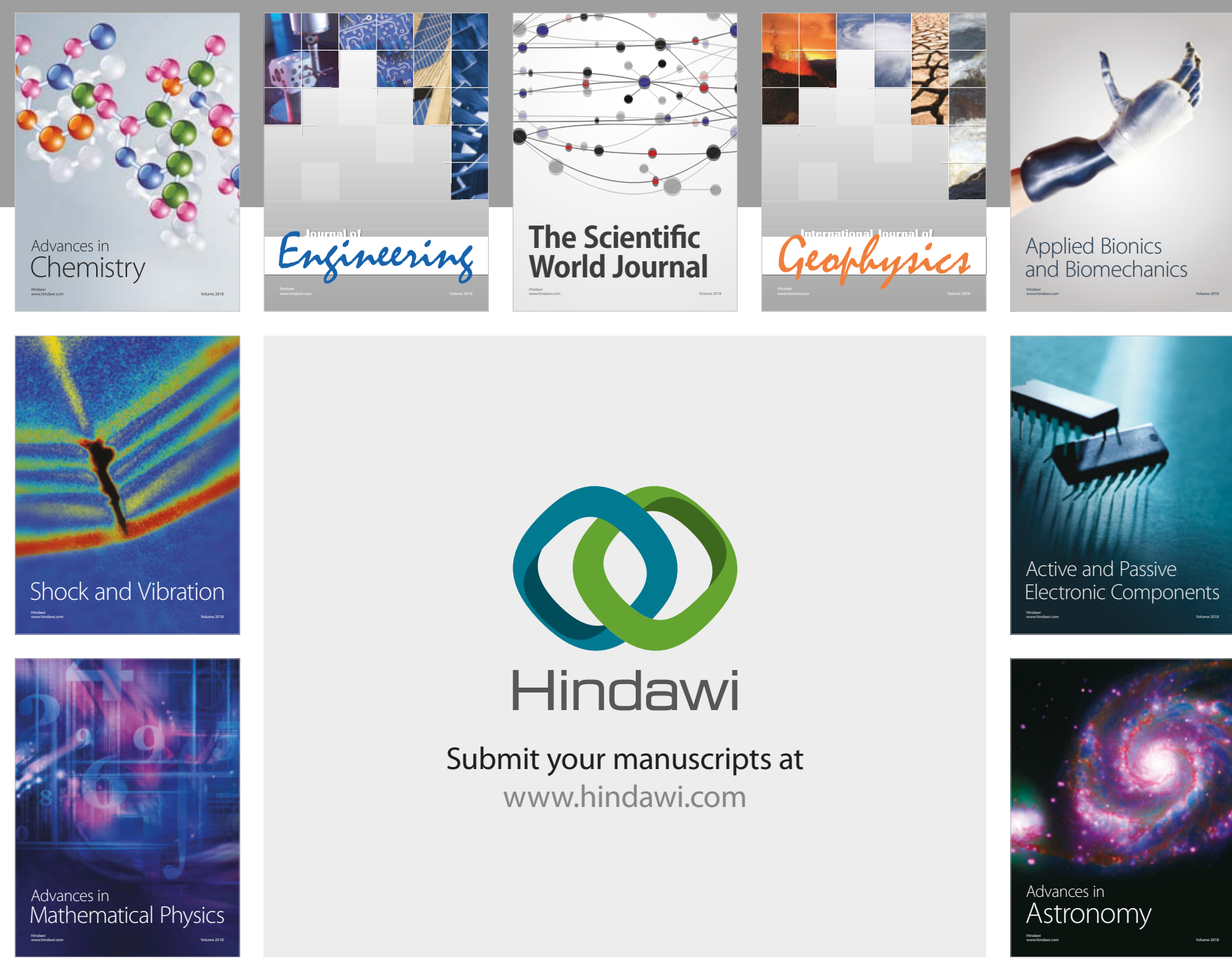

Submit your manuscripts at

www.hindawi.com

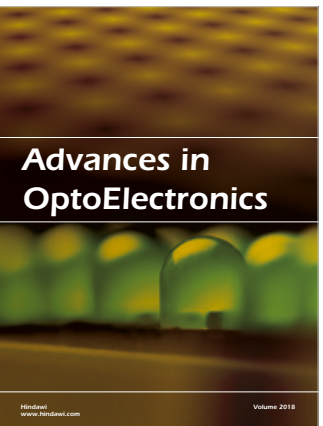

\section{Rotcting Machinery}
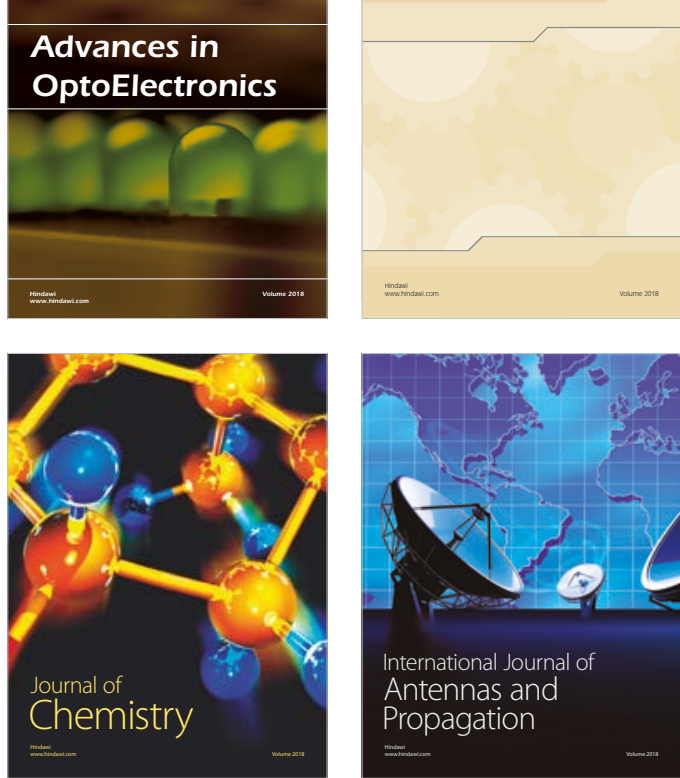

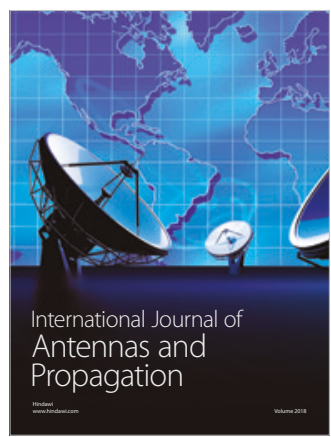

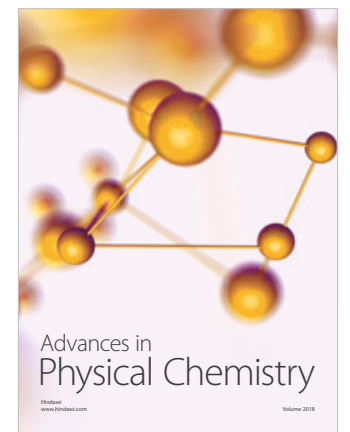

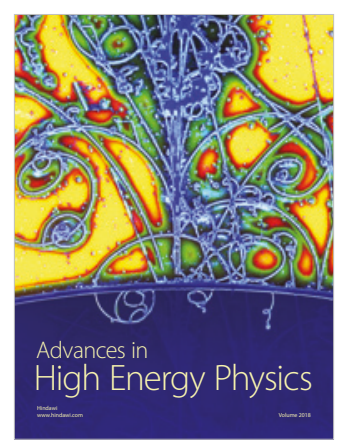

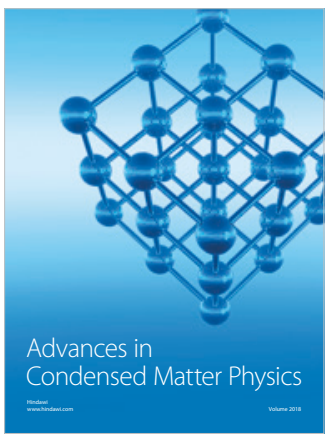

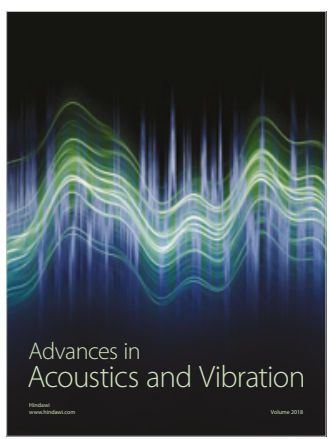

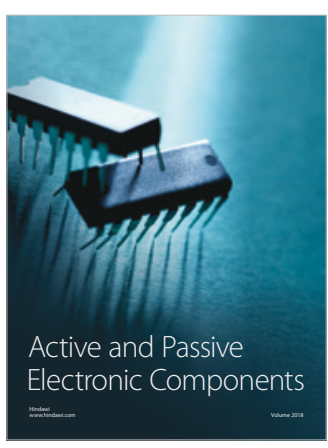
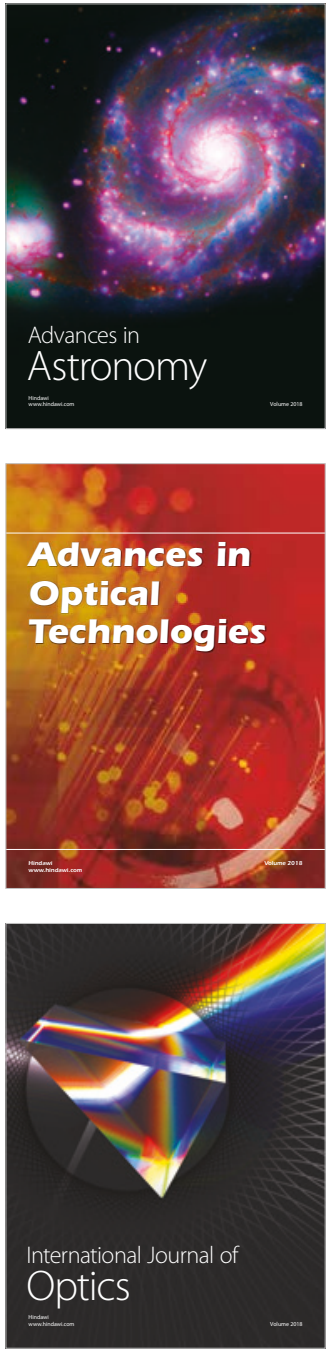